\title{
Pensando en la integración de los posgrados centroamericanos: metodología investigativa aplicada en la Confederación Universitaria Centroamericana
}

\section{Thinking about the Integration of Postgraduate Studies Methodological proposal for the Central American University Confederation.}

\author{
Mario Alejandro Morúa Saborío ${ }^{1}$ \\ Universidad Estatal a Distancia, Costa Rica
}

\begin{abstract}
Resumen. La tesis doctoral que origina el presente artículo metodológico lleva por título La integración regional de los estudios de posgrado ofrecidos por las universidades públicas en Centroamérica y República Dominicana. El propósito de este artículo es exponer el método y la metodología implementados en dicha tesis. El tema de la tesis fue desarrollado desde el paradigma de investigación cualitativo y con enfoque de gestión. Previo a la definición del método por seguir para su desarrollo, se efectuaron dinámicas orientadas a problematizar las principales ideas que giran en torno a la integración centroamericana de los estudios de posgrado. Esta integración constituyó el objeto de estudio. El tipo general del método que se eligió para el desarrollo de la tesis doctoral en mención es el hermenéutico-dialéctico, el cual se caracteriza fundamentalmente por considerar la forma natural desde la cual las personas comprenden e interpretan significados. Este método sigue la lógica que va de lo general a lo particular y viceversa, sin distorsionar la fragmentación del "todo" en las partes que lo conforman. Más bien, identifica, de manera consciente, en esa comprensión e interpretación de significados, la existencia de las complejas interrelaciones que surgen entre el "todo" y sus partes constitutivas, pero a la vez, entre estas consigo mismas y con el "todo" que conforman. La puesta en marcha de este método se hizo por medio de tres técnicas: 1) la entrevista semiestructurada, 2) la observación participante y 3) la revisión documental, lo que permitió la correspondiente triangulación para analizar los datos e información obtenida.
\end{abstract}

Palabras clave. Integración regional, educación superior, globalización, investigación, metodología.

\begin{abstract}
The doctoral thesis that gives rise to this methodological article is entitled "The Regional Integration of Postgraduate Studies Offered by Public Universities in Central America and the Dominican Republic" (Morúa, 2016). The purpose of this article is to expose the method and methodology implemented in this thesis. The subject of this thesis was developed from the paradigm of qualitative research and management approach. Prior to the definition of the method to be followed for its development, it was necessary to carry out dynamics aimed at problematizing the main ideas that revolve around the Central American integration of postgraduate studies as an object of study. The general type of the method proposed for the development of the doctoral thesis mentioned above, which will be widely discussed in this article, is the hermeneutic-dialectic, which is fundamentally characterized by considering the natural form from which people understand and interpret meanings, following the logic that goes from the general to the particular and vice versa, without distorting the fragmentation of the "Whole" in the parts that comprise it, but rather, consciously identifying in that understanding and interpretation of meanings, the existence of the complex interrelations that arise between the "Whole" and its constituent parts, but at the same time between these with themselves and in turn with the "Whole" they form. The implementation of this method was done through three techniques: a) the semi-structured interview, b) the participant observation and c) the documentary review, which allowed the corresponding triangulation to analyze the data and information obtained.
\end{abstract}

Keywords. Regional integration, higher education, globalization, research, methodology.

${ }^{1}$ Mario Alejandro Morúa Saborío. Doctor en Administración. Profesor del Sistema de Estudios de Posgrado e investigador asociado en el Programa de Investigación en Fundamentos de Educación a Distancia (PROIFED), ambos de la Universidad Estatal a Distancia de Costa Rica. Dirección electrónica: mmorua@uned.ac.cr 


\section{Introducción}

La globalización es un fenómeno tan antiguo y complejo como la misma humanidad y los modelos sociales desde los cuales esta ha sido definida $y$, a la vez, ha evolucionado en todas las áreas que la determinan.

La internacionalización se ha constituido en una respuesta a ese fenómeno, en muchas de las actividades que desarrolla la humanidad... la educación superior universitaria, en términos generales $\mathrm{y}$, específicamente, en el contexto regional centroamericano -que es el contexto en el cual se orientará el abordaje de este artículono escapa a esa realidad.

El enfoque temático, tanto del presente artículo como de la tesis de la cual se deriva, es propio de la gestión académico-administrativa que se realiza para los estudios de posgrado, por parte de las universidades públicas que los imparten en la Región y que forman parte de la Confederación Universitaria Centroamericana (CUC). Por lo tanto, es conveniente en este punto definir dos conceptos inmersos en esa gestión académico-administrativa: por un lado, el de educación superior; $y$, por otro lado, el de internacionalización de la educación superior.

En primera instancia, se define educación superior, esta comprende el periodo de formación con mayores exigencias académicas y científicas, que ocurre previo al inicio de una carrera profesional por parte de las personas que ingresan a esta. Por lo tanto, es en ese lapso de formación superior que las personas adquieren experiencias de naturaleza científica, académica, técnica y profesional, esenciales para la comprensión teórica y metodológica del saber (Morúa, 2016, pp. 111-112; Ulrich, 2006, p. 30). Así lo explica Ulrich (2006): “Entre los alcances de la educación superior, pueden citarse la investigación y la enseñanza, es decir, la creación de conocimiento (investigación), así como la transferencia y preservación de éste (enseñanza)" p.30, "lo cual, necesaria e inevitablemente por su propósito y naturaleza académica, involucra directamente a los estudios de posgrado, especialmente maestrías tanto profesionales como académicas y doctorados" (Morúa, 2016, pp. 111-112).

En el contexto centroamericano y del Caribe, la educación superior nace a partir de esquemas europeos, particularmente de aquellos provenientes de España. Con respecto a sus inicios, en el año 1538, se fundó la Universidad Autónoma de Santo Domingo, en República Dominicana, primera universidad de América. Ciento cuarenta y tres años después, en el año 1681, se fundó en Guatemala la Universidad de San Carlos de Guatemala, primera casa de estudios superiores centroamericanos.

$\mathrm{Al}$ alcanzar la independencia en el año 1821, por parte de cada una de las antiguas provincias que conformaron la Capitanía General de Guatemala, ya como Estados soberanos, estas iniciaron sus propias dinámicas de desarrollo y de consolidación de la educación superior (Morúa, 2016, pp.10-12).

Ahorabien, elconceptodeinternacionalización, en la historia y en el contexto de la educación superior, según Romero (2002) "se refiere a la posición asumida por las universidades ante el inminente fenómeno que ha caracterizado a la humanidad desde hace miles de años: la globalización..." (pp. 97-98), fenómeno ampliamente caracterizado "por los medios académicos, políticos y empresariales... sin que haya consenso sobre el significado de dicho término. En realidad, el concepto de globalización tiene connotaciones de tipo ideológico, socioeconómico, político y cultural" (Romero, 2002, pp. 97-98).

Desde esta perspectiva, "surge entonces una dimensión particular del fenómeno conocido 
como globalización, que es la dimensión referente a la globalización del conocimiento, con implicaciones directas en la gestión académico-administrativa que se hace desde las universidades públicas en la región" (Morúa, 2016, p. 73); "las ventajas comparativas de los países ya no se basan en la dotación de recursos naturales, la mano de obra barata o la ubicación geográfica, sino en el desarrollo del talento humano... A este proceso ha contribuido en forma notoria la llamada revolución de la información" (Romero, 2002, pp. 103-105).

Esa revolución ha propiciado $-y$ lo continúa haciendo-el surgimiento de grandes volúmenes de datos; por lo tanto, también se crea el cuestionamiento sobre la diferencia entre dato, información y conocimiento, y se genera "una especie de sobresaturación de información, al punto que en la actualidad el problema del conocimiento depende no tanto de si tenemos o no acceso a la información, sino de la forma en que hagamos uso de ella" (Romero, 2002, pp. 103105). Al respecto, este mismo autor explica que:

el conocimiento requiere de cierto grado de razonamiento y enjuiciamiento que organiza la información mediante su comparación y clasificación. Para ello es necesario un ejercicio interactivo sujeto-objeto del conocimiento, en el cual se debe asumir una posición crítica y creativa, con el propósito no solo de apropiarnos de la información disponible, sino, además, y sobre esta base, generar nuevo conocimiento (Romero, 1999, pp. 27-28; Romero, 2002, pp. 103-105).

Así pues, "dentro de ese contexto de la globalización del conocimiento, como un fenómeno mundial e inminente en el cual se encuentra inmersa la educación superior, emerge la masificación, regulaciones e internacionalización como reformas de la educación superior en América Latina y el Caribe" (Rama, 2006, p. 17).
Con respecto a los desafíos, tanto para la educación superior en general como para los estudios de posgrados en específico, particularmente, los ofrecidos por las universidades públicas en la región, autores como Romero (2002, pp. 103-105) y Rama (2006, p. 17) proponen los siguientes:

1. Capacidad para diseñar y gestionar redes interuniversitarias regionales en el nivel de posgrado.

2. Capacidad para integrar y convalidar programas de posgrado en el nivel regional.

3. Movilización e intercambio de docentes y estudiantes en el nivel regional.

4. Compartir buenas prácticas en formación, contenidos, metodologías, entre las universidades miembros de la región.

En respuesta a ese complejo contexto que surge como parte del fenómeno de la globalización en general $\mathrm{y}$, específicamente, en lo referente a la globalización del conocimiento -contexto que continuamente "genera nuevas dinámicas globales y locales (glocales)" (Rama, 2006, p. 17) - se crea en la región, en el año 1948, y como parte de las dinámicas de internacionalización regional de la educación superior universitaria, la Confederación Universitaria Centroamericana (CUC) y su máxima autoridad: el Consejo Superior Universitario Centroamericano (CSUCA) (Morúa, 2016, p. 12).

Actualmente, la CUC se integra por las siguientes veintiún universidades públicas por país (Morúa, 2016, pp. 187-188):

Guatemala:

1) Universidad de San Carlos de Guatemala (USAC).

Belice:

1) Universidad de Belice (UB). 
El Salvador:

Universidad de El Salvador (UES).

\section{Honduras:}

1) Universidad Nacional Autónoma de Honduras (UNAH), 2) Universidad Pedagógica Nacional Francisco Morazán (UPNFM) y 3) Universidad Nacional de Agricultura (UNAG).

Nicaragua:

1) Universidad Nacional Autónoma de Nicaragua-Managua (UNAN-Managua), 2) Universidad Nacional Autónoma de NicaraguaLeón (UNAN-León), 3) Universidad Nacional de Ingeniería (UNI) y 4) Universidad Nacional Agraria de Nicaragua (UNA).

Costa Rica:

1)Universidad de Costa Rica (UCR), 2) Instituto Tecnológico de Costa Rica (ITCR), 3) Universidad Nacional (UNA), 4) Universidad Estatal a Distancia (UNED) y 5) Universidad Técnica Nacional de Costa Rica (UTN).

Panamá:

1) Universidad de Panamá (UP), 2) Universidad Tecnológica de Panamá (UTP), 3) Universidad AutónomadeChiriquí(UNACHI),4)Universidad Especializada de las Américas (UDELAS) y 5) Universidad Marítima Internacional de Panamá (UMIP).

\section{República Dominicana:}

1) Universidad Autónoma de Santo Domingo (UASD).

Estas universidades públicas ofrecen carreras en los distintos campos del saber, en los niveles de pregrado, grado y posgrado. Sobre el origen de los estudios de posgrado en el mundo, se cita lo siguiente:

Los primeros cursos de posgrado aparecieron en los Estados Unidos a comienzos del siglo XX bajo la denominación de máster, y buscaban prolongar y completar los estudios de su breve ciclo universitario regular, de tan solo cuatro años, frente a los cinco o seis de las universidades europeas.

Uno de los hallazgos más interesantes logrados hasta ahora en la investigación sobre estudios avanzados es la identificación de los cinco modelos dominantes (el alemán, el norteamericano, el francés, el inglés y el soviético), y la constatación de las demás experiencias son simples casos o variaciones de tales modelos. Ello es un indicador interesante acerca de los vínculos existentes entre el posgrado y los programas de colonialismo y dependencia económico-política que dominan la historia contemporánea (Morales, 1997) (López, 2002, pp.65-66).

"En relación con ese surgimiento de los estudios de posgrado en la región, desde finales del siglo pasado y hasta la actualidad, los mismos han sido y continúan siendo impartidos por universidades, tanto públicas como privadas" (Morúa, 2016, pp. 111-112); en otras palabras, son parte inherente del quehacer académico-administrativo de dichas casas de estudios superiores, no un apéndice de estas con fines distintos a los delegados por mandato constitucional de cada país, mandato sustentado por la contribución del erario público.

El inicio de los programas de posgrado en la región data, aproximadamente, desde la penúltima y última década del siglo $\mathrm{XX}$. Aunque existen algunos registros de estudios de posgrados que iniciaron en décadas anteriores, sobre todo en Costa Rica y en Guatemala.

Entre el año 2005 y el 2015, la Región experimentó un incremento del $57 \%$ en la cantidad de Maestrías y Doctorados ofrecidos por las veintiuna universidades públicas que actualmente conforman la CUC, pasando de 680 programas de posgrado en el año 2005, a 1065 en el año 2015, sin contar especialidades médicas (Morúa, 2016, p. 110). 
Tal incremento numérico, en un contexto mundial y regional altamente dinámico, ha significado, a la vez, el incremento en la complejidad inherenteen losesquemas diseñados para la gestión académico-administrativa de dichos estudios de posgrados. Asimismo, esto evidencia la necesidad de reflexionar sobre la posibilidad de diseñar esquemas y modelos de gestión más novedosos que incorporen como parte de su diseño o estructura esa complejidad.

"El primer encuentro de investigación y posgrado, que organizó y realizó el Sistema Centroamericano de Investigación y Posgrado (SICAR) del CSUCA en el año 2012, en la Universidad de El Salvador" (Morúa, 2016, p. 16) fue un espacio donde el Consejo de Directores Posgrados compartió algunas de las principales deficiencias y limitaciones comunes o compartidas, en cuanto a la gestión académico-administrativa de los estudios de posgrado en la región.

Esas deficiencias y limitaciones estuvieron relacionadas "con: planificación, gestión, impacto, integración, comunicación, entre otras" (Morúa, 2016, pp. 16-17), lo que permitió implementar la primera premisa metodológica que fue agruparlas en las siguientes dimensiones: planificación estratégica, autoevaluación y acreditación, gestión presupuestaria, gestión del talento humano, internacionalización y comunicación. De esta manera, se formuló la siguiente pregunta o problema en la tesis doctoral mencionada al principio del presente artículo:

¿Cómoafectanlas deficienciasylimitaciones de la actual gestión académico-administrativa en los sistemas de estudios de posgrado de las universidades miembros de la Confederación, la integración y articulación de los elementos que conforman las dimensiones referentes a planificación estratégica, autoevaluación y acreditación, gestión presupuestaria, gestión del talento humano, internacionalización y comunicación? (Morúa, 2016, p. 26).

\section{Metodología}

Para efectos del presente artículo, así como los de este apartado, la metodología resultante, utilizada para desarrollar la investigación doctoral de la cual se deriva este artículo y que ampliamente será compartida en el siguiente apartado referente a los "Resultados", tuvo su origen en la observación participante in situ, en cuatro países de la región: 1) Costa Rica (en dos momentos diferentes); 2) Honduras; 3) Panamá (en dos momentos diferentes); y 4) Nicaragua (en dos momentos diferentes), durante siete lapsos diferentes, además de la documentación de datos e información obtenidos a partir de dicha observación.

Esos datos e información fueron los insumos básicos que propiciaron los espacios para el desarrollo de dinámicas orientadas a problematizar las principales ideas que giran en torno a la integración centroamericana de los estudios de posgrado. Dicha integración se reconoció como el objeto de estudio. Una vez formulado y planteado el problema a partir de la observación, así como los datos e información recabados con esta, conjuntamente con las dinámicas orientadas a la problematización, se definieron el enfoque y el método de investigación que se siguió para desarrollar la investigación doctoral. A su vez, esto condujo al diseño metodológico de técnicas e instrumentos con los cuales se continuó recabando datos e información en función de los objetivos también formulados en las primeras fases de desarrollo de la investigación doctoral ya mencionada.

\section{Resultados}

Como principal resultado metodológico, se afirma que el diseño y el abordaje metodológico implementado para el desarrollo de la investigación doctoral, de la cual se deriva este 
artículo, se hizo desde el "método hermenéutico dialéctico", el cual:

es un método general de comprensión y la interpretación es el modo natural de conocer de los seres humanos. La hermenéutica tiene como misión descubrir los significados de las cosas, interpretar lo mejor posible las palabras, los escritos, los textos y los gestos, así como cualquier acto $\mathrm{u}$ obra, pero conservando su singularidad en el contexto del que forma parte.

De acuerdo con la lógica dialéctica, las partes son comprendidas desde el punto de vista del todo. Dilthey (1900) llamó círculo hermenéutico a este proceso interpretativo, es decir, al movimiento que va del todo a las partes y de las partes al todo tratando de buscarle sentido (Gurdian, 2007, pp. 147-149).

Se utilizó dicho método fundamentalmente por dos razones:

1. Es afín con la forma natural en que las personas otorgan significancia a las cosas, sin perder de vista el valor que cada una de esas cosas tiene dentro del conjunto en el cual forma parte.

2. El método mantiene también estrecha afinidad con los enfoques teóricos desde los cuales se abordó la investigación doctoral en la correspondiente fundamentación conceptual, donde tan importante es el análisis y comprensión de cada uno de los elementos que conforman el todo, como las interrelaciones que definen las dinámicas secuenciales, paralelas y simultáneas que surgen entre cada uno de esos elementos y el todo del cual también forman parte. (Morúa, 2016, pp. 95-96)

Además, tanto el método de investigación seguido como la metodología aplicada, ambos desde el enfoque cualitativo de investigación, permitieron hacer una adecuada triangulación de datos e información. Tales datos fueron recopilados y procesados a partir de las técnicas e instrumentos diseñados y provenientes de los siguientes tres tipos de fuentes: el criterio experto, la documentación existente y generada durante el proceso investigativo; y por último, la observación cualitativa in situ, sobre las cuales más adelante se brindará mayor detalle.

Ahora bien, al tratarse este artículo justamente sobre la metodología resultante aplicada para dar respuesta a la pregunta o al problema planteado en la tesis doctoral, seguidamente, se hará mención de los once aspectos que integraron dicha metodología. Estos aspectos serán explicados y desarrollados, con amplitud, en el apartado "Resultados" del artículo, ya que el resultado de este artículo será, precisamente, ese diseño metodológico que permitió la recopilación de datos e información, los cuales luego fueron procesados y analizados en función del tema central de la tesis doctoral.

Esos once aspectos son:1) tipo de investigación, 2) sujetos de información, 3) población de estudio, 4) criterios de selección, 5) personas expertas entrevistadas, 6) categorías de análisis, 7) técnicas e instrumentos para la recolección de datos e información, 8) procedimiento general para la recolección de información, 9) análisis de datos e información, 10) criterios de validez, 11) principios éticos que guiaron el proceso de investigación.

\section{Tipo de investigación}

En relación con el tipo de investigación, esta es aplicada "porque es investigación dirigida hacia la solución de problemas prácticos y específicos en áreas delineadas" (Eyssautier, 2007, p. 114). A la vez, es descriptiva-exploratoria "por cuanto presenta la información tal cual es, con el fin de describir la situación como se presenta al momento de realizar la investigación, procurando conclusiones intermedias; o bien, tentativas que respalden los procesos de toma de decisiones" (Schmelkes, 2004, pp. 34-35). Además, el desarrollo de la investigación se hizo 
desde el enfoque o paradigma cualitativo, por lo que se denotan las siguientes características:

1. La naturaleza de los datos es cualitativa (textos, narraciones, significados, etcétera).

2. Datos profundos y enriquecedores. En inglés: soft.

3. La recolección de los datos está orientada a proveer de un mayor entendimiento de los significados y experiencias de las personas. El investigador es el instrumento de recolección de los datos, se auxilia de diversas técnicas que se desarrollan durante el estudio. Es decir, no se inicia la recolección de los datos con instrumentos preestablecidos, sino que el investigador comienza a aprender por observación y descripciones de los participantes y concibe formas para registrar los datos que se van refinando conforme avanza la investigación.

4. El análisis varía dependiendo del modo en que hayan sido recolectados los datos.

5. Análisis fundamentado en la inducción analítica.

6. Uso moderado de la estadística (conteo, algunas operaciones aritméticas).

7. Análisis basado en casos o personas y sus manifestaciones.

8. Análisis simultáneo a la recolección de los datos.

9. El análisis consiste en describir información y desarrollar temas.

10. Datos en forma de texto, imágenes, piezas audiovisuales, documentos y objetos personales. (Morúa, 2016, pp. 94-95; Hernández, Fernández y Baptista, 2010, p. 13).

\section{Sujetos de información}

Conrespectoalossujetosdeinformación,fueron aquellas personas nombradas como directoras, decanas o coordinadoras de programas de posgrados en alguna universidad miembro de la Confederación, o bien, personas que en algún momento ocuparon u ocupan, al momento de realizar esta investigación doctoral, el cargo de Rectoría o Vicerrectoría de Investigación, Académica o Docencia en alguna de estas Universidades miembros de la Confederación Universitaria Centroamericana (Morúa, 2016, p. 96).

\section{Población de estudio}

En relación con la población de estudio, estuvo constituida por personas con formación en diferentes campos del saber, con nivel de posgrado, mínimo con maestría profesional, o bien, máximo con doctorado, y que ocupan u ocuparon puestos además de docencia e investigación, con perfiles directivos o al menos de coordinación en algunas de las universidades miembros de la Confederación (Morúa, 2016, p. 96).

En cuanto a las personas expertas, se entrevistaron: "1) Directores(as) de áreas ácadémicas, 2) Coordinadores(as) del CDSICAR (ahora CD-SIRCIP) y 3) Miembros de la Comisión Técnica del ahora SIRCIP" (Morúa, 2016, p. 96).

\section{Criterios de selección}

Tanto para informantes como para expertos se fijaron los siguientes criterios de selección:

1. Anuencia y disposición para participar en esta investigación.

2. Ocupar ohaber ocupadoen el pasado reciente (no más de diez años) un cargo de autoridad en la gestión académico-administrativa de los programas de posgrado.

3. Tener al menos el grado académico de Magíster (Mag.) o Magister Scientiae (M.Sc.) (Morúa, 2016, pp. 96-97)

Personas expertas entrevistadas

Un total de seis personas expertas; fue entrevistado: tres de Panamá, una de Guatemala, 
una de Honduras y una de Costa Rica, las seis del género masculino. Se trató de coordinar citas para entrevistar a personas expertas del género femenino tanto en Costa Rica como en otros países de la Región; sin embargo, ninguna de ellas confirmó disponibilidad de tiempo para atender a dicha entrevista en el periodo establecido para tal fin, incluso una de estas personas del género femenino y de Costa Rica se negó rotundamente a ser entrevistada (Morúa, 2016, p. 97). Otros datos e información importante sobre este aspecto son los siguientes:

De las seis personas entrevistadas, cinco habían obtenido el diploma de doctor y una el diploma de maestría. Además, una de estas personas al momento de la entrevista ocupaba desde hacía 3 años el puesto de Vicerrector de Investigación y Posgrado y las otras cinco personas ocupaban el puesto de decano o director de sus respectivos estudios de posgrado, con una antigüedad en ese puesto entre los 2 y 9 años. Cuatro de estas personas al momento de la entrevista se ubicaron en un rango de edad entre los 51 y los 60 años, una se ubicó en menos de 40 años y otra en más de 61.

Estas seis personas a las que entrevisté, además de cumplir con todos los criterios de selección establecidos en la "metodología aplicada" para esta investigación doctoral, eran -al momento de la entrevista- representantes activos ante el CSUCA de sus respectivas universidades miembros de la Confederación, lo que además de confirmar la pertinencia del criterio experto emitido por cada una de ellas, brinda un mayor peso a esteen dicho análisis. (Morúa, 2016, p. 97).

\section{Categorías de análisis}

Con el fin de brindar una adecuada fundamentación teórica, sobre la cual apoyar el abordaje analítico de los datos e información recabados en la investigación doctoral, se hizo una categorización y subcategorización de temas, a partir de las dimensiones incluidas en la pregun- ta o problema formulado para la investigación doctoral (Morúa, 2016, p. 26).

El concepto "categoría" es una palabra relacionada con la idea de clases o series que tienen características comunes; por lo tanto, las categorías estarían sujetas a clasificación, o bien, a "categorizaciones"; "surgen o derivan directamente de los datos que representan fenómenos, los cuales son ideas analíticas pertinentes". (Morúa, 2016, p.98; Romero, 2005, pp. 113-118).

En consecuencia, para la investigación, se estableció la siguiente categorización y subcategorización:

Categoría: administración de la educación superior pública. Subcategoría: políticas (universidades miembros de la Confederación).

Categoría: calidad de la educación superior. Subcategorías: eficiencia-eficacia, autoevaluación, acreditación internacional e innovación.

Categoría: planificación para el desarrollo. Subcategorías: planificación estratégica, modelos de gestión, administración-gestión (talento humano, presupuestaria, etc.), ejecución y evaluación.

Categoría: internacionalización de la educación superior pública. Subcategorías: capacidad de diseñar y gestionar redes interuniversitarias regionales a nivel de posgrado, capacidad de integrar y convalidar programas de posgrado a nivel regional, movilización e intercambio de docentes y estudiantes a nivel regional, compartir buenas prácticas en formación, contenidos, metodologías, entre las universidades miembros de la confederación.

Categoría: comunicación. Subcategorías: sistema de comunicación intra e interuniversitario a nivel de posgrados, eficiencia y eficacia de la comunicación.

Categoría: cambio organizacional.Subcategorías: clima organizacional y acción gerencial. (Morúa, 2016, pp. 99-100). 
Técnicas e instrumentos para la recolección de datos e información

Con respecto a las técnicas e instrumentos implementados para la recolección de datos e información, en la tabla 1, se ofrece el detalle correspondiente.

La observación cualitativa se realizó in situ durante siete momentos diferentes que ocurrieron entre los años 2013 y 2016, en diferentes países de la región donde se utilizó, como guía de observación cualitativa, las agendas y los programas de trabajo propuestos para cada uno de esos momentos. De esta manera, posteriormente, sería posible contrastar lo observado con el problema, los objetivos, las categorías y las subcategorías propuestas para esta investigación doctoral. (Morúa, 2016, pp. 102-103).

En relación con la guía para realizar la entrevista semiestructurada a personas expertas, estuvo

Tabla 1

Técnicas para la recolección de información

\begin{tabular}{|c|c|c|}
\hline Técnica & Definición & Instrumento \\
\hline Observación cualitativa & $\begin{array}{l}\text { No es mera contemplación ("sentar- } \\
\text { se a ver el mundo y tomar notas"); } \\
\text { nada de eso, implica adentrarnos } \\
\text { en profundidad a situaciones so- } \\
\text { ciales y mantener un papel activo, } \\
\text { así como una reflexión permanente. } \\
\text { Estar atento a los detalles, sucesos, } \\
\text { eventos e interacciones. (Hernán- } \\
\text { dez, Fernández, Baptista, 2008) }\end{array}$ & Guía para observación cualitativa \\
\hline Entrevistas semiestructuradas & $\begin{array}{l}\text { Las entrevistas semiestructuradas, } \\
\text { por su partes, se basan en una guía } \\
\text { de asuntos o preguntas y el entre- } \\
\text { vistador tiene la libertad de intro- } \\
\text { ducir preguntas adicionales para } \\
\text { precisar conceptos u obtener mayor } \\
\text { información sobre los temas desea- } \\
\text { dos (es decir, no todas las pregun- } \\
\text { tas están predeterminadas) (Her- } \\
\text { nández et al., 2008) }\end{array}$ & $\begin{array}{l}\text { Guía para realizar la entrevista } \\
\text { semiestructurada }\end{array}$ \\
\hline $\begin{array}{l}\text { Documentos, registros, materiales } \\
\text { y artefactos, individuales o } \\
\text { grupales }\end{array}$ & $\begin{array}{l}\text { Una fuente muy valiosa de datos } \\
\text { cualitativos son los documentos, } \\
\text { materiales y artefactos diversos. } \\
\text { Nos pueden ayudar a entender el } \\
\text { fenómeno central de estudio. (Her- } \\
\text { nández et al., 2008) }\end{array}$ & $\begin{array}{l}\text { Documentación disponible en me- } \\
\text { dios físicos y electrónicos }\end{array}$ \\
\hline
\end{tabular}

Fuente. Metodología de la investigación (Morúa, 2016, pp.101-102; Hernández, Fernández, Baptista, 2008, pp. 587, 597, 614). 
integrada por los siguientes cinco apartados: a) problema de investigación, b) objetivo general, c) objetivo específico uno, d) objetivo específico dos y e) objetivo específico tres; cada uno de esos apartados con preguntas relacionadas, específica y directamente, con un grupo de categorías o subcategorías de análisis, de tal manera que ninguna de estas fuera omitida. (Morúa, 2016, pp. 193-201).

La documentación disponible en medios físicos y electrónicos que se utilizó fue aquella alta- mente relacionada con la temática, especializada y disponible tanto en bibliotecas como bases de datos, en formato impreso o digital, así como las actas o memorias y documentación generada en los seis momentos mencionados anteriormente en la descripción de la observación cualitativa.

Procedimiento general para la recolección de información

Este procedimiento queda descrito en la siguiente tabla 2.

Tabla 2

Procedimiento general para la recolección de datos e información

Fases del procedimiento

Fase 1. Exploratoria/reflexiva y de diseño de la investigación

Fase 2. Entrada en el escenario

\section{Descripción de acciones}

Diseño del proyecto, construcción teórica del problema de estudio.

Fase 3. Selección de las personas participan- Primera entrevista con el grupo de informantes sobre tes: el propósito de la investigación, la factibilidad de su participación, así como la obtención del consentimiento informado (Anexo 2). Contacto con los expertos y las expertas.

Fase 4. Recolección de la información

Aplicación de las estrategias para la recolección de la información: grabación, transcripción de entrevistas, anotaciones de observación participante, transcripción de entrevistas a expertos y expertas.

Fase 5. Retirada del escenario Se realizará la retirada del campo, que incluirá un adecuado cierre del proceso y agradecimiento por parte del investigador.

Fase 6. Análisis a profundidad de los datos Se procederá al análisis cualitativo (hermenéutico) de y preparación del informe final los datos y a la preparación del informe final.

Fuente. Datos de investigación doctoral 


\section{Análisis de datos e información}

Con respecto al procedimiento seguido para el análisis de los datos e información recabada, este se detalla en la tabla 3:

\section{Tabla 3}

Fases del procedimiento para el análisis de datos e información

\begin{tabular}{l} 
Fases del procedimiento \\
\hline Recolección de los datos (fase interactiva con las \\
fases de: 1) codificación de las unidades: primer \\
nivel y 2) describir las categorías codificadas \\
que emergieron del primer nivel) y simultánea \\
con la codificación de las categorías: segundo \\
nivel \\
Organización de los datos e información
\end{tabular}

Preparar los datos para el análisis

Revisión de los datos (lectura y observación)

Descubrir la(s) unidad(es) de análisis.

Codificación de las unidades: primer nivel (fase interactiva con la fase de recolección de los

datos)

Describir las categorías codificadas que emergieron del primer nivel (fase interactiva con la fase de recolección de los datos)

Codificación de las categorías: segundo nivel (fase simultánea con la fase de recolección de los datos)

\section{Descripción de acciones}

Entrevistas.

Observaciones.

Anotaciones y registros.

Determinar criterios de organización.

Organizar los datos de acuerdo con los criterios.

Limpiar grabaciones de ruidos, digitalizar, imágenes, filtrar videos.

Transcribir datos verbales en texto (incluyendo bitácoras y anotaciones).

Obtener un panorama general de los materiales.

Elegir cuál es la unidad de análisis o significado adecuada, a la luz de la revisión de los datos.

Localizar unidades y asignarles categorías y códigos.

Conceptualización.

Definiciones.

Significados.

Ejemplos

Agrupar categorías codificadas en temas y patrones. Relacionar categorías.

Ejemplificar temas, patrones y relaciones de análisis.

\section{GENERAR TEORÍAS, HIPÓTESIS, EXPLICACIONES}

Fuente. Datos de investigación doctoral 
Adicionalmente, para apoyar el análisis de datos e información, se implementó el "Análisis DAFO-CAME", el cual es una variación del clásico análisis F.O.D.A., con la incorporación del análisis C.A.M.E; se invierten las letras del primero para 1) proponer un orden sobre los aspectos que deben atenderse y 2) brindar una propuesta coherente para el abordaje ordenado de prioridades. (Morúa, 2016, p.106). Esto se ilustra en la tabla tabla 4.

Tabla 4

Análisis DAFO-CAME

\begin{tabular}{ccc}
\hline Ordenamiento & D.A.F.O. & C.A.M.E. \\
$1 .^{\circ}$ & D: debilidades & C: corregir \\
$2 .^{\circ}$ & A: amenazas & A: afrontar \\
$3 .^{\circ}$ & F: fortalezas & M: mantener \\
$4 .^{\circ}$ & O: oportunidades & E: explotar \\
\hline
\end{tabular}

Fuente. Tesis doctoral (Morúa, 2016, p.106).

En las siguientes figuras 1, 2 y 3, se explica la lógica que se siguió con este tipo de análisis.

Figura 1. Siguiendo el sentido de las manecillas del reloj, a partir del recuadro "Debilidades", se mencionan y describen cada uno de los componentes considerados para realizar el análisis D.A.F.O. La cruz con punta de fechas indica que el análisis debe ser integral. Es tomada de la tesis La Integración regional de los estudios de posgrado ofrecidos por las universidades públicas en Centroamérica y República Dominicana por Morúa Saborío, M. A., 2016, p. 106.

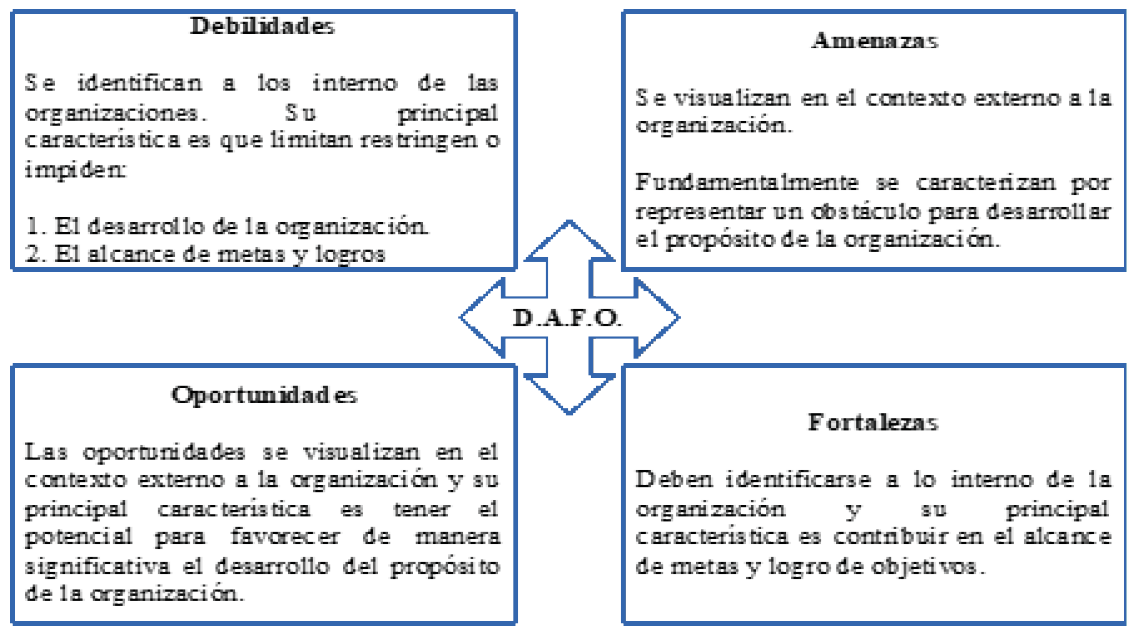

Figura 1. Componentes considerados para el análisis D.A.F.O. 


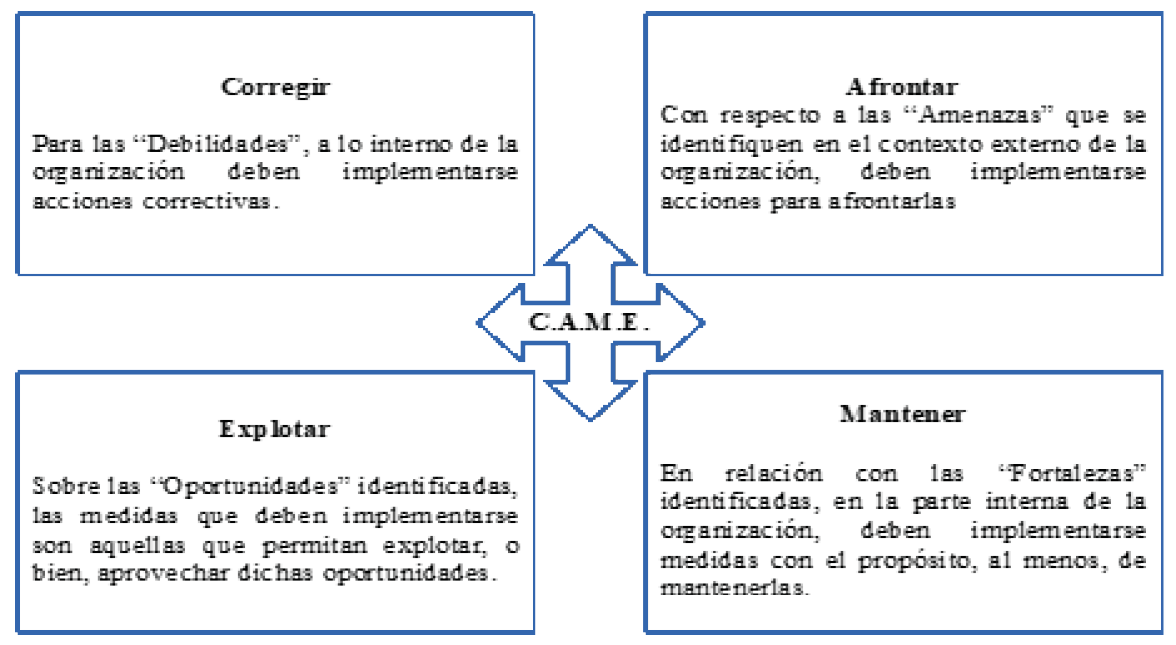

Figura 2. Componentes considerados para el análisis C.A.M.E.

Figura 2. Siguiendo el sentido de las manecillas del reloj, a partir del recuadro "Corregir", se mencionan y describen cada uno de los componentes considerados para realizar el análisis C.A.M.E. La cruz con punta de fechas indica que el análisis debe ser integral. Es tomada de la tesis La integración regional de los estudios de posgrado ofrecidos por las universidades públicas en Centroamérica y República Dominicana por Morúa Saborío, M. A., 2016, p. 107.

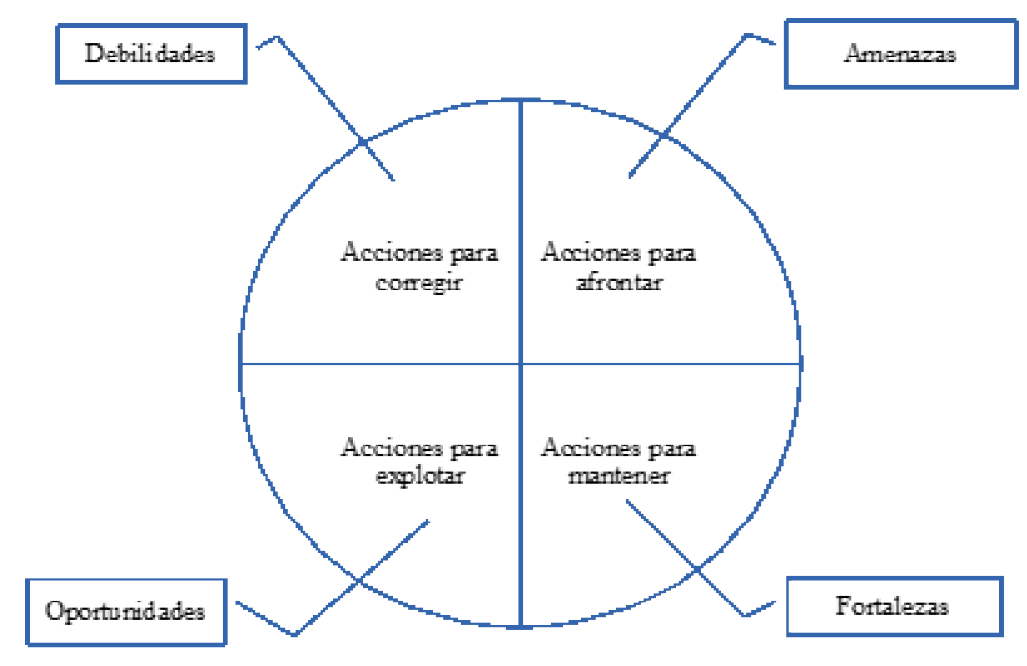

Figura 3. Integración de los componentes para el análisis D.A.F.O.-C.A.M.E. 
Figura 3. Una vez más, siguiendo el sentido de las manecillas del reloj, a partir del recuadro "Debilidades", se integran los componentes tanto para realizar el análisis D.A.F.O. como el C.A.M.E. En dicho análisis, corresponde a las debilidades, acciones por corregir; a las amenazas, acciones por afrontar; a las fortalezas, acciones por mantener; y las oportunidades, acciones por explotar. Es tomada de la tesis La integración regional de los estudios de posgrado ofrecidos por las universidades públicas en Centroamérica y República Dominicana por Morúa Saborío, M.A., 2016, p. 107.

\section{Criterios de confiabilidad y validez}

Con respecto a la confiabilidad cualitativa, esta "se denomina dependencia o consistencia lógica..." a la vez, afirman que es "como el grado en que diferentes investigadores que recolecten datos similares en el campo y efectúen los mismos análisis, generen resultados equivalentes..." (Morúa, 2016, p. 108; Hernández, Fernández y Baptista, 2008, p. 662).

En cuanto a la credibilidad o validez interna cualitativa, "Se refiere a si el investigador ha captado el significado completo y profundo de las experiencias de los participantes, particularmente de aquellas vinculadas con el planteamiento del problema..." (Morúa, 2016, p. 108; Hernández, et al., 2008, pp. 665-666).

Principios éticos que guiaron el proceso de investigación

Al ser una actividad con gran demanda intelectual, y por ende humana, la investigación en general debe considerar el componente ético en todos sus alcances. Particularmente para la investigación doctoral de la cual se derivó este artículo, "fueron consideradas todas las buenas costumbres conocidas para la actividad investigativa, particularmente aquellas asociadas a los valores de: Rigor académico, Respeto y Transparencia" (Morúa, 2016, p.109).
Ese componente ético de la investigación se tradujo en la implementación del consentimiento informado; para ello, se diseñó un formulario que fue entregado a cada una de las personas entrevistadas, para su respectiva lectura, firma y devolución, a fin de su respectiva custodia. En dicho formulario, se brindó explicación y descripción exhaustivas sobre la problemática, los objetivos, los alcances y las limitaciones en dicha investigación, así como los medios para contactar a las personas directamente involucradas, en caso que surgieran dudas.

\section{Conclusión}

Como conclusiones resultantes a partir de la exposición realizada en el presente artículo, puntualmente, se comparten a continuación las siguientes:

1. El artículo ofrece un acercamiento preciso al método y metodología implementados para recabar, procesar y analizar los datos e información sobre el objeto de estudio abordado en la tesis doctoral de la cual se deriva esta pesquisa, en función de los correspondientes marcos referencial, teórico y metodológico de dicha tesis, así como del enfoque disciplinar al que pertenece.

2. Considerando la complejidad del objeto de estudio y su contexto, así como las características del método hermenéutico-dialéctico, este permite mantener estrecha coherencia y consistencia en cada una de las fases de desarrollo de la investigación doctoral con la cual se abordó el objeto de estudio, también, en función de los correspondientes marcos referencial, teórico y metodológico de esta.

3. El método y metodología implementados se orientan a generar conocimiento aplicable en áreas específicas y previamente delineadas dentro del campo disciplinar de las ciencias de la administración en general; y específicamente, en 
aquellas áreas estrechamente relacionadas con el objeto de estudio.

4. El perfil de los sujetos de información, de la población de estudio y de las personas expertas, así como los criterios de selección predefinidos, contribuye significativamente a mantener rigurosidad en cuanto a la confiabilidad y validez del dato.

5. La categorización y su correspondiente subcategorización conducen a la clasificación sistemática de las principales ideas y análisis relacionados con el objeto de estudio.

6. Por medio de las técnicas de investigación aplicadas, con su respectiva instrumentación, se lograla triangulaciónnecesaria, loquecontribuye, con la confiabilidad y validez, al dato como tal.

7. Tanto el procedimiento para la recolección de datos e información como para su análisis ofrece una forma esquematiza y ordenada para el desarrollo de ambas fases, lo cual significa mayor control en la ejecución de estas.

8. El análisis D.A.F.O.-C.A.M.E., ampliamente utilizado para el abordaje de casos en el campo de la administración, principalmente en lo referente a "dirección organizacional", se implementó con el fin de articular las técnicas e instrumentos clásicos de la investigación académica desde el paradigma cualitativo, por ejemplo: la observación, la entrevista y la revisión documental, con el enfoque de gestión desde el cual fue abordado el objeto de estudio. De esta manera, fue posible identificar, con mayor pertinencia, aquellas debilidades, amenazas, fortalezas y oportunidades relacionadas con dicho objeto; pero a la vez, para identificar, igualmente de forma pertinente, posibles acciones por corregir, por afrontar, por mantener y por explotar, respectivamente.

9. Los principios éticos que guiaron el proceso de investigación ofrecen transparencia y rigor ético. Este artículo se presenta de tal forma que personas relacionadas con el tema y miembros de diferentes comunidades académicas puedan tener acceso a este, con el fin, al menos, de valorar la aplicabilidad del método y metodología en este $\mathrm{u}$ otros casos, así como para implementar variaciones orientadas hacia la mejora de ellos.

\section{Referencias}

Eyssautier, M. (2007). Metodología de la Investigación. 5.a edición. México: Thomson.

Gurdian, F. A. (2007). El paradigma cualitativo en la investigación socioeducativa. Costa Rica: Colección IDER, Coordinación Educativa y Cultural Centroamericana (CECC) de la Agencia Española de Cooperación Internacional (AECI).

Hernández, S. R.; Fernández, C. C. y Baptista, L. P. (2008). Metodología de la investigación. México: McGraw-Hill.

Hernández, S.R.; Fernández, C.C.y Baptista, L.P. (2010). Metodología de la investigación. México: McGraw-Hill.

López, R. (2002). Los estudios de postgrado en el mundo. Revista de Enseñanza Universitaria, 20, 65-74. Recuperado de http://institucional.us.es/revistas/universitaria/20/art 5.pdf

Morúa Saborío, M. A. (2016). La integración regional de los estudios de posgrado ofrecidos por las universidades públicas en Centroamérica y República Dominicana (tesis doctoral). Universidad Estatal a Distancia, San José-Costa Rica.

Rama Vitale, C. (2006). La tercera reforma de la educación superior en América Latina y el Caribe: masificación, regulaciones e internacionalización. Revista Educación y Pedagogía, 46(18), 11-24. Recuperado de http:// aprendeenlinea.udea.edu.co/revistas/index. php/revistaeyp/article/viewFile/6875/6292

Romero,A. (2002). Globalización y pobreza.Universidad de Nariño, Colombia: E-libro.net. 
Romero, Ch., C. (2005). La categorización un aspecto crucial en la investigación cualitativa. Revista de Investigaciones Cesmag, 11, 113-118.

Schmelkes, C. (2004). Manual para la presentación de anteproyectos e informes de investiga- ción (tesis). 2.a edíción. México: Oxford University Press.

Ulrich, T. (2006). Reformas de los modelos de laeducaciónsuperioren Europa, Japón y América Latina: análisis comparado. Argentina: Miño y Dávila.

Recibido: : 17 de enero de 2017

Aceptado: 29 de setiembre de 2017 